\title{
Single-photon laser spectroscopy of cold antiprotonic helium
}

\author{
Masaki Hori ${ }^{1}$ \\ Published online: 24 October 2018 \\ (C) The Author(s) 2018
}

\begin{abstract}
Some laser spectroscopy experiments carried out by the Atomic Spectroscopy and Collisions Using Slow Antiprotons (ASACUSA) collaboration to measure the single-photon transition frequencies of antiprotonic helium $\left(\bar{p} \mathrm{He}^{+} \equiv \bar{p}+\mathrm{He}^{2+}+e^{-}\right)$atoms are reviewed. The $\bar{p} \mathrm{He}^{+}$were cooled to temperature $T=1.5-1.7 \mathrm{~K}$ by buffer-gas cooling in a cryogenic gas target, thus reducing the thermal Doppler width in the single-photon resonance lines. The antiproton-to-electron mass ratio was determined as $M_{\bar{p}} / m_{e}=1836.1526734(15)$ by comparisons with the results of three-body quantum electrodynamics calculations. This agreed with the known proton-to-electron mass ratio.
\end{abstract}

Keywords Antiprotonic helium $\cdot$ CPT symmetry $\cdot$ Exotic atoms

\section{Introduction}

Metastable antiprotonic helium $\left(\bar{p} \mathrm{He}^{+} \equiv \bar{p}+\mathrm{He}^{2+}+e^{-}\right)$is a three-body atom composed of a helium nucleus, an electron in the ground state, and an antiproton occupying a Rydberg state of principal and orbital angular momentum quantum numbers $n \sim \ell-1 \sim 38$ [1-3]. By measuring its transition frequencies by laser spectroscopy [4-7], and comparing the values with the results of three-body quantum electrodynamics (QED) calculations, the antiproton-to-electron mass ratio $M_{\bar{p}} / m_{e}$ can in principle be determined with a relative precision of $<10^{-11}$. This corresponds to the best determinations of the proton-to-electron mass ratio $M_{p} / m_{e}$ from Penning trap experiments [8-12], or laser spectroscopy of $\mathrm{HD}^{+}$ molecular ions [13-15]. Spectroscopy of $\bar{p} \mathrm{He}^{+}$also provides a consistency test of CPT symmetry, which may be complementary to the experiments on antihydrogen atoms [16$18]$. The data have also been used to constrain exotic fifth forces that may exist at the $\sim 1$ Å length scale [19-23].

This article is part of the Topical Collection on Proceedings of the 13th International Conference on Low Energy Antiproton Physics (LEAP 2018) Paris, France, 12-16 March 2018

Edited by Paul Indelicato, Dirk van der Werf, and Yves Sacquin

Masaki Hori

Masaki.Hori@mpq.mpg.de

1 Max-Planck-Institut für Quantenoptik, Hans-Kopfermann-Strasse 1, 85748 Garching, Germany 
The atoms are experimentally synthesized via the reaction, $\bar{p}+\mathrm{He} \rightarrow \bar{p} \mathrm{He}^{+}+e^{-}$, which occurs when an antiproton beam [24] is allowed to come to rest in a helium gas target [25-27]. The transition frequencies of $\bar{p} \mathrm{He}^{+}$spanning the infrared to ultraviolet regions have been calculated [1-3] to a relative precision of $\sim 10^{-10}$ by evaluating the QED corrections up to order $m_{e} \alpha^{7}$ in atomic units. Here $m_{e}$ and $\alpha$ respectively denote the electron mass and the fine structure constant. The calculations used the International Council for Science Committee on Data for Science and Technology (CODATA) 2010 recommended values of the fundamental constants [28], which included the fine structure constant $\alpha$, the ${ }^{3} \mathrm{He}$ - and ${ }^{4} \mathrm{He}$-to-electron mass ratios, the Bohr radius, and the Rydberg constant. The corrections to the atomic transition frequencies that arise from the finite charge radii of the helium nucleus (4 to $7 \mathrm{MHz})$ and of the antiproton $[29,30](<1 \mathrm{MHz})$ are small in the case of $\bar{p} \mathrm{He}^{+}$, because the spatial overlap between the Rydberg antiproton orbital and the nucleus is relatively small, and because the negatively-charged antiproton is polarized away from the $1 s$ electron in the atom. Past experiments on antiproton-nucleon scattering and $\mathrm{X}$-ray spectroscopy of antiprotonic atoms provide an upper limit on the radius of antiprotons [31-34], but the $\bar{p} \mathrm{He}^{+}$experiments have not yet reached a precision that is sensitive to this.

\section{Buffer gas cooling of $\bar{p} \mathrm{He}^{+}$atoms}

In the experimental target, the thermal motions of $\bar{p} \mathrm{He}^{+}$at temperature $T$ broadens the width of the measured single-photon laser resonances by a factor, $v \sqrt{8 k_{B} T \log 2 / M c^{2}}$. Here $v$ denotes the transition frequency, $k_{B}$ the Boltzmann constant, $M$ the atom's mass, and $c$ the speed of light. In past experiments, the corresponding loss in the spectral resolution limited [35] the precision in the determination of the resonance centroid to around $10^{-7}-10^{-8}$. One way to reach a precision beyond this Doppler limit was recently provided by twophoton spectroscopy $[4,36]$, in which the $\bar{p} \mathrm{He}^{+}$was irradiated by two counterpropagating ultraviolet laser beams.

An alternative method of increasing the spectral resolution involved cooling some $2 \times 10^{9}$ $\bar{p} \mathrm{He}^{+}$atoms to a temperature $T=1.5-1.7 \mathrm{~K}$, by allowing the $\bar{p} \mathrm{He}^{+}$to undergo elastic collisions with cryogenic helium gas [5]. This cooling is in contrast to the behavior seen in other types of hadronic exotic atoms, such as pionic hydrogen [37], which was found to be heated by collisions with the surrounding $\mathrm{H}_{2}$ molecules that led to the deexcitation of the atom. The density of the buffer gas ( $T \sim 1.5 \mathrm{~K}$ and $P=40-170 \mathrm{~Pa})$ was carefully adjusted so that the $\bar{p} \mathrm{He}^{+}$atoms, once formed, rapidly underwent a few hundred or more cooling collisions. The atoms were then interrogated by the resonant laser beam. The presence of the $1 s$ electron protected a significant fraction of the $\bar{p} \mathrm{He}^{+}$from annihilation during this cooling.

The experiment (see Fig. 1) was carried out by utilizing the pulsed beam of the Antiproton Decelerator (AD) of CERN that contained between $2 \times 10^{7}$ and $3 \times 10^{7}$ antiprotons with a kinetic energy $E=5.3 \mathrm{MeV}$ and repetition rate $f=0.01 \mathrm{~Hz}$. A fraction of the antiprotons were slowed down to $E=75 \mathrm{keV}$ by allowing them to traverse a $3 \mathrm{~m}$ long radiofrequency quadrupole decelerator. The device was also recently used to attempt to measure the annihilation cross sections of antiprotons that traversed some thin target foils [38-42]. The 75-keV antiprotons that emerged from the RFQD were transported by a beamline and allowed to enter the cryogenic helium gas target. The target was in thermal contact with an open-cycle Joule-Thomson cryocooler which was cooled to a temperature $T \sim 1.3 \mathrm{~K}$. The $\bar{p} \mathrm{He}^{+}$atoms were irradiated by $\Delta t=40$ to $100 \mathrm{~ns}$ long laser pulses [43] with peak powers $P=0.5$ to 

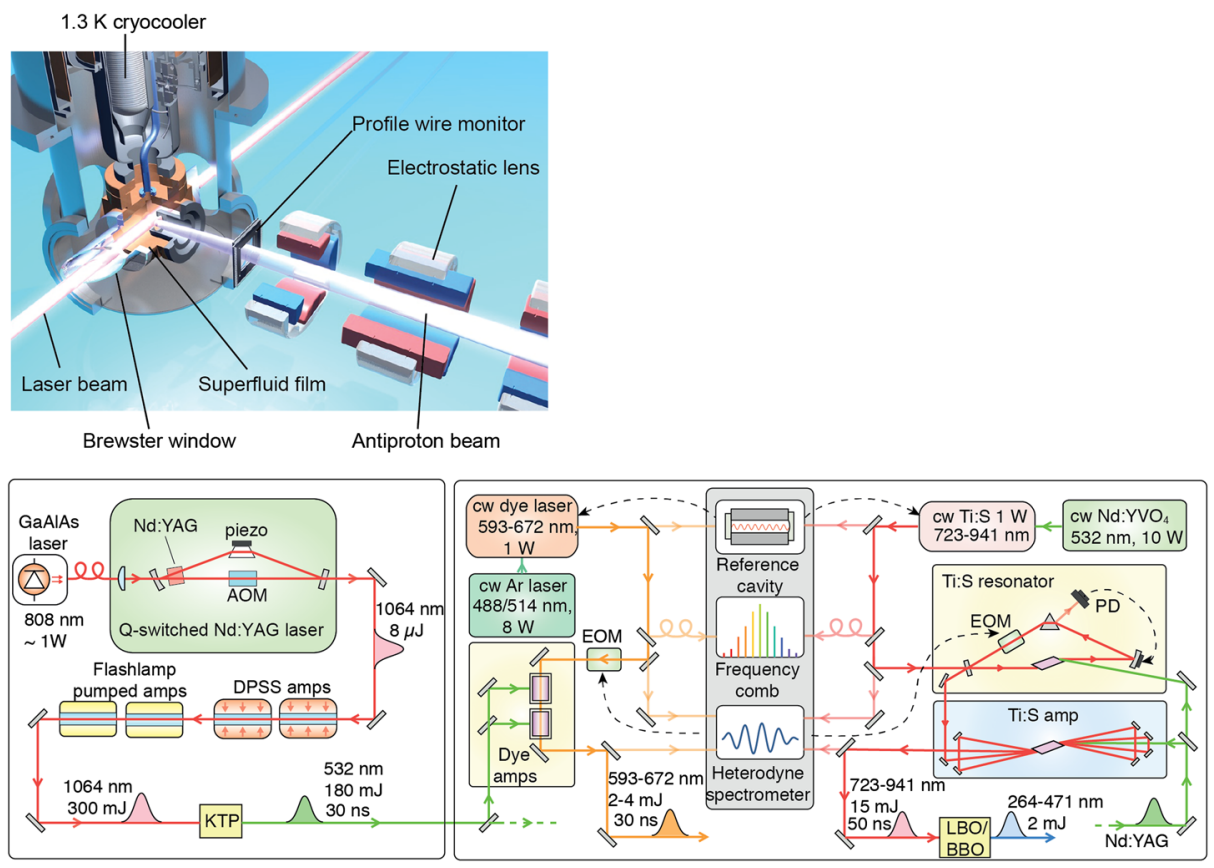

Fig. 1 Experimental layout [5] used to synthesize $\bar{p} \mathrm{He}^{+}$, which are then cooled to temperature $T=1.5-1.7$ $\mathrm{K}$ (top). Laser system used for single-photon spectroscopy (bottom)

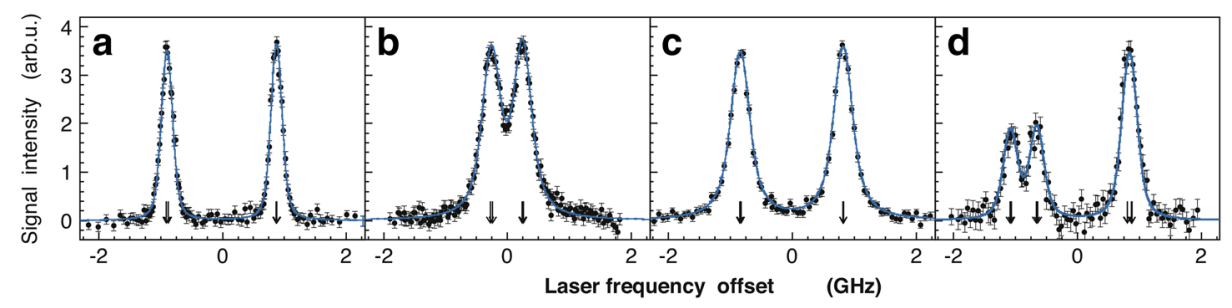

Fig. 2 The resonance profiles of the single-photon transitions $\mathbf{a}(n, \ell)=(37,35) \rightarrow(38,34), \mathbf{b}(n, \ell)=$ $(39,35) \rightarrow(38,34)$, and $\mathbf{c}(n, \ell)=(38,35) \rightarrow(39,34)$ of buffer-gas cooled $\bar{p}^{4} \mathrm{He}^{+}$. d The resonance profile $(36,34) \rightarrow(37,33)$ of $\bar{p}^{3} \mathrm{He}^{+}$. The $x$-abscissa indicates the offset of the laser frequency relative to the resonance centroid. Solid curves indicate the best fit of an ab initio model based on the optical Bloch equation. From Ref. [5]

$10 \mathrm{~kW}$ and wavelengths $\lambda=264$ to $841 \mathrm{~nm}$, which were generated by Ti:Sapphire or dye laser systems.

The spectra of Fig. 2a-c show the resonance profiles of the transitions $(n, \ell)=$ $(37,35) \rightarrow(38,34),(39,35) \rightarrow(38,34)$, and $(38,35) \rightarrow(39,34)$ of $\bar{p}^{4} \mathrm{He}^{+}$. The spectra were obtained by plotting the intensities of the antiproton annihilation signals which were induced at multiple laser frequencies between -1 and $1 \mathrm{GHz}$ around the resonance centroid. The arrows indicate the positions of the four hyperfine sublines that arise from the spin-spin interaction between the antiproton and electron in $\bar{p}^{4} \mathrm{He}^{+}$. The single photon resolution of some of these profiles exceeds those of sub-Doppler two-photon spectroscopy experiments 
using higher-temperature atoms [4]; this indicates the low $T=1.5-1.7 \mathrm{~K}$ temperature of the atoms. Figure $2 \mathrm{~d}$ shows the profile of the $\bar{p}^{3} \mathrm{He}^{+}$resonance $(n, \ell)=(36,34) \rightarrow(37,33)$; the three-peak structure arises from the eight unequally spaced hyperfine sublines caused by the interactions between the ${ }^{3} \mathrm{He}$ nuclear, electron, and antiproton spins. For each transition, the spin-independent transition frequencies were determined by fitting the profiles with a theoretical line shape (indicated by blue lines). These line shapes were obtained by solving the optical Bloch equations [36] that model the corresponding single-photon transition. The experimental uncertainties in the determinations of the $\bar{p} \mathrm{He}^{+}$frequencies include contributions from the statistical uncertainty $( \pm 1 \mathrm{MHz})$ that arises from the limited number of measured atoms, and the systematic uncertainty of $0.4-3 \mathrm{MHz}$ caused by the fitting function. The dye and Ti:Sapphire lasers used to excite the atoms contain a spurious frequency modulation, which were measured with a precision of 0.4-1.0 MHz. The ac Stark effects that were induced by the lasers, and magnetic Zeeman shifts due to residual magnetic fields, caused systematic effects of $<0.1 \mathrm{MHz}$ and $<0.2 \mathrm{MHz}$, respectively.

Eight transition frequencies of $\bar{p}^{4} \mathrm{He}^{+}$atoms and five transition frequencies of $\bar{p}^{3} \mathrm{He}^{+}$ were measured with relative uncertainties between $2.5 \times 10^{-9}$ and $15 \times 10^{-9}$. The experimental frequencies $v_{\text {exp }}$ (Fig. 3a, open circles with error bars) agree with theoretical $v_{\text {th }}$ values (filled squares). Due to the cooling techniques and improvements in the experimental apparatus, this agreement is a factor of 1.4 to 10 times better than previous single-photon experiments [35] of $\bar{p} \mathrm{He}^{+}$. The uncertainties for most of the theoretical frequencies $v_{\text {th }}$ arise from uncalculated QED contributions of orders higher than $m_{e} \alpha^{7}$.

When the antiproton-to-electron mass ratio $M_{\bar{p}} / m_{e}$ used in the QED calculations was changed by a fractional amount $1 \times 10^{-9}$, the theoretical $\bar{p} \mathrm{He}^{+}$frequencies $v_{\text {th }}$ changed by $2.6 \times 10^{-9}$ to $2.7 \times 10^{-9}$. By minimizing the difference between the frequencies $v_{\text {exp }}$ and $v_{\text {th }}$, the mass ratio was determined as, $M_{\bar{p}} / m_{e}=1836.1526734(15)$. The one-standard deviation uncertainty in the parenthesis includes the three contributions $9 \times 10^{-7}, 11 \times 10^{-7}$, and $3 \times 10^{-7}$ of the experimental statistical and systematic uncertainties, and the theoretical uncertainty, respectively.

The atomic mass of the electron has been determined [11] with a relative precision of $3 \times 10^{-11}$ in an experiment involving the confinement of a ${ }^{12} \mathrm{C}^{5+}$ ion in a Penning trap. The cyclotron frequency of its motion in a magnetic field and the precession frequency of the electron spin was measured, and the results compared with QED calculations of its $g$-factor [44]. From this and the proton mass which was recently measured in a separate measurement with a Penning trap [12] from the same collaboration, the proton-to-electron mass ratio was determined as, $M_{p} / m_{e}=1836.152673346(81)$. In Fig. 3b, these latest $M_{p} / m_{e}$ mass ratios are shown together with the results of previous experiments that were determined by comparing the cyclotron frequencies of protons and electrons in a Penning trap [8], laser spectroscopy of cold $\mathrm{HD}^{+}$molecular ions [13], and the CODATA 2010 recommended value [28]. The recent high-precision values are in good agreement with the $M_{\bar{p}} / m_{e}$ ratio determined from $\bar{p} \mathrm{He}^{+}$. The TRAP and BASE experiments of CERN have compared the cyclotron frequencies of antiprotons and $\mathrm{H}^{-}$ion pairs confined in a Penning trap [45, 46]. A limit of $5 \times 10^{-10}$ was set $[5,47]$ on any deviation between the antiproton and proton masses and charges by combining the results with the $\bar{p} \mathrm{He}^{+}$spectroscopic data.

The $\bar{p}^{4} \mathrm{He}^{+}$transition $(n, \ell)=(40,36) \rightarrow(41,35)$ was recently studied by laser spectroscopy [6]. For this a stimulated first-order Raman scattering process in a $\mathrm{H}_{2}$ gas cell was 

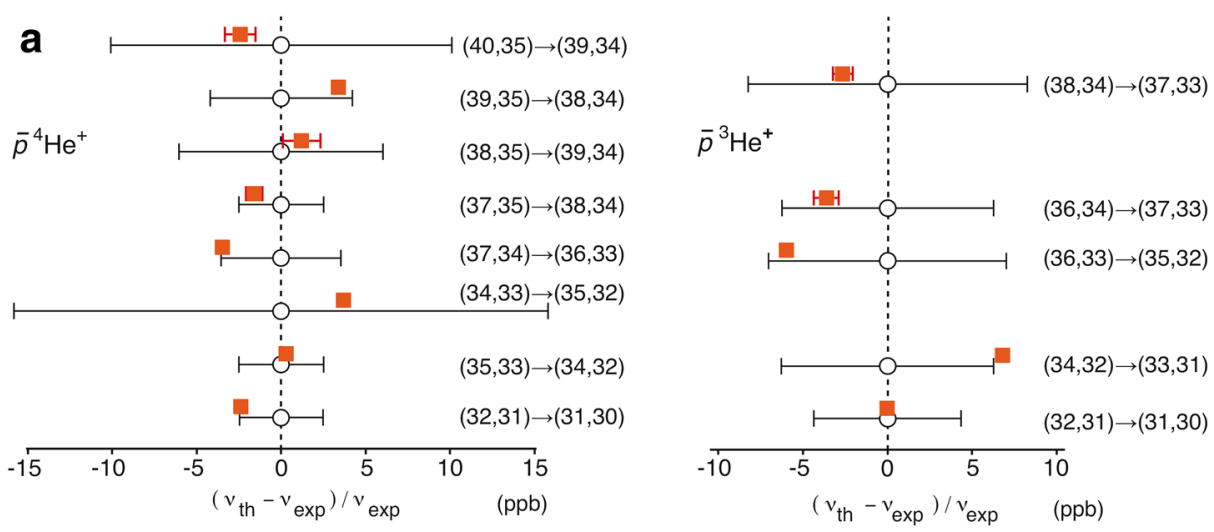

b

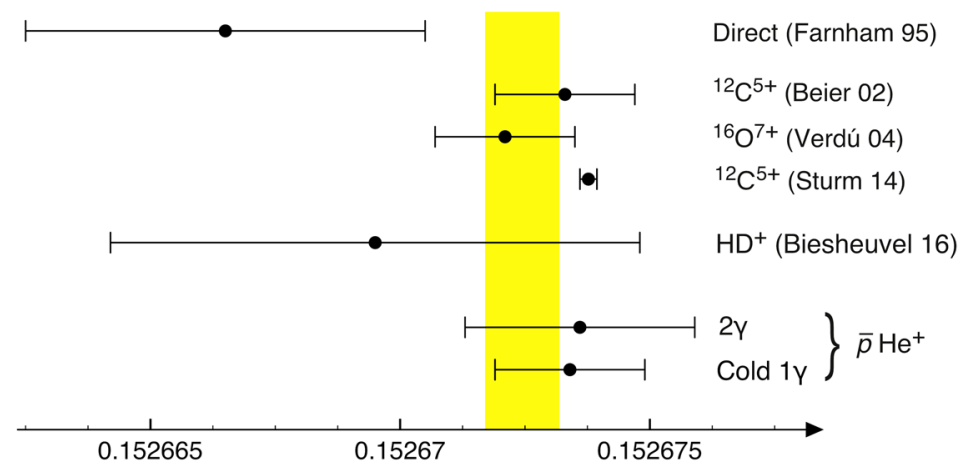

(Anti)proton-to-electron mass ratio -1836

Fig. 3 a Comparison of experimental and calculated transition frequencies, showing the fractional differences between the experimental (open circles) and theoretical (squares) values of 13 transition frequencies of cooled $\bar{p}^{4} \mathrm{He}^{+}$and $\bar{p}^{3} \mathrm{He}^{+}$atoms. b Proton-to-electron mass ratios measured in Penning traps and laser spectroscopy of $\mathrm{HD}^{+}$molecular ions, compared with the antiproton-to-electron mass ratio determined by spectroscopy of $\bar{p} \mathrm{He}^{+}$. The shaded area represents the CODATA 2010 recommended value [28]. The more precise CODATA 2014 value is similar to the high-precision result of Ref. [11], see text. From Ref. [5]

used to generate the $7 \mathrm{~ns}$ long laser pulses of wavelength $\lambda=1154.9 \mathrm{~nm}$ needed to excite the transition. The experiments showed that most of the metastable populations are concentrated into states of principal quantum number $n \geq 40$, whereas the states $n>41$ contain relatively small populations of antiprotons. A laser-microwave-laser triple resonance method was used to study the hyperfine structure of the $(n, \ell)=(36,34)$ state of $\bar{p}^{3} \mathrm{He}^{+}$[7].

\section{Conclusions and future perspectives}

The transition frequencies of antiprotonic helium cooled to temperature $T \sim 1.5-1.7 \mathrm{~K}$ were measured to a fractional precision between $2.5 \times 10^{-9}$ and $16 \times 10^{-9}$ by single photon spectroscopy. The precision on the calculations of $\bar{p} \mathrm{He}^{+}$energies are now more than an order of magnitude higher than these experimental uncertainties, whereas further improvements in the theoretical precision are expected in the next 5 years. The Extra Low Energy 
Antiproton Ring (ELENA) facility due to begin full operation in 2021 will provide a highquality, cooled antiproton beam of kinetic energy $E=100 \mathrm{keV}$. Using this beam, samples of $\bar{p} \mathrm{He}^{+}$could be formed in a smaller volume in the experimental target, so that lasers of lower power and higher spectral resolution could be utilized. The superior intensity and positional stability of the antiproton beam should improve the signal-to-noise ratio on the $\bar{p} \mathrm{He}^{+}$spectral lines measured by the experiment. This may allow us to resolve weak $\bar{p} \mathrm{He}^{+}$ transitions between metastable states of smaller natural width, which can be measured with a higher precision.

Metastable pionic helium $\left(\pi \mathrm{He}^{+}\right)$is an analogous three-body atom [48-50] that consists of a helium nucleus, an electron in the $1 s$ ground state, and a negative-charged pion in a Rydberg state of quantum numbers $n \sim \ell-1 \sim 16$. The spectral lines of these atoms have never been directly observed, and so the existence of $\pi \mathrm{He}^{+}$is so far hypothetical. Laser spectroscopy of the atom is currently being attempted at the $590 \mathrm{MeV}$ ring cyclotron facility of the Paul Scherrer Institute. The $\pi^{-}$mass [51] can in principle be determined with a fractional precision of $10^{-8}$ to $10^{-6}$, by comparing the experimental frequencies with those derived from QED calculations. The first experiments have been carried out and data analysis is ongoing.

Acknowledgements Open access funding provided by Max Planck Society. We are deeply indebted to the ASACUSA collaboration and to the CERN PS and AD operation teams. We thank V.I. Korobov. This work was supported by the Max-Planck-Gesellschaft.

Open Access This article is distributed under the terms of the Creative Commons Attribution 4.0 International License (http://creativecommons.org/licenses/by/4.0/), which permits unrestricted use, distribution, and reproduction in any medium, provided you give appropriate credit to the original author(s) and the source, provide a link to the Creative Commons license, and indicate if changes were made.

\section{References}

1. Korobov, V.I., Hilico, L., Karr, J.-P.: $m \alpha^{7}$-order corrections in the hydrogen molecular ions and antiprotonic helium. Phys. Rev. Lett. 112, 103003 (2014)

2. Korobov, V.I., Hilico, L., Karr, J.-P.: Theoretical transition frequencies beyond $0.1 \mathrm{ppb}$ accuracy in $\mathrm{H}_{2}^{+}$, $\mathrm{HD}^{+}$, and antiprotonic helium. Phys. Rev. A 89, 032511 (2014)

3. $\mathrm{Hu}, \mathrm{M} .-\mathrm{H}$. et al.: Variational calculation of energy levels for metastable states of antiprotonic helium. Chem. Phys. Lett. 654, 114 (2016)

4. Hori, M. et al.: Two-photon laser spectroscopy of antiprotonic helium and the antiproton-to-electron mass ratio. Nature 475, 484 (2011)

5. Hori, M. et al.: Buffer-gas cooling of antiprotonic helium to 1.5 to $1.7 \mathrm{~K}$, and antiproton-to-electron mass ratio. Science 354, 610 (2016)

6. Kobayashi, T. et al.: Observation of the $1154.9 \mathrm{~nm}$ transition of antiprotonic helium. J. Phys. B: At. Mol. Opt. Phys. 46, 245004 (2013)

7. Friedreich, S. et al.: Microwave spectroscopic study of the hyperfine structure of antiprotonic ${ }^{3} \mathrm{He}$. J. Phys. B: At. Mol. Opt. Phys. 46, 125003 (2013)

8. Farnham, D.L., Van Dyck, R.S. Jr., Schwinberg, P.B.: Determination of the electron's atomic mass and the proton/electron mass ratio via Penning trap mass spectroscopy. Phys. Rev. Lett. 75, 3598 (1995)

9. Beier, T. et al.: New Determination of the Electron's Mass. Phys. Rev. Lett. 88, 011603 (2002)

10. Verdú, J. et al.: Electronic $g$ factor of hydrogenlike oxygen ${ }^{16} \mathrm{O}^{7+}$. Phys. Rev. Lett. 92, 093002 (2004)

11. Sturm, S. et al.: High-precision measurement of the atomic mass of the electron. Nature 506, 467 (2014)

12. Heiße, F. et al.: High-precision measurement of the proton's atomic mass. Phys. Rev. Lett. 119, 033001 (2017)

13. Biesheuvel, J. et al.: Probing QED and fundamental constants through laser spectroscopy of vibrational transitions in $\mathrm{HD}^{+}$. Nat. Commun. 7, 10385 (2016) 
14. Koelemeij, J.C.J. et al.: Vibrational spectroscopy of $\mathrm{HD}^{+}$with 2-ppb accuracy. Phys. Rev. Lett. 98, 173002 (2007)

15. Alighanbari, S., Hansen, M.G., Korobov, V.I., Schiller, S.: Rotational spectroscopy of cold and trapped molecular ions in the Lamb-Dicke regime. Nat. Phys. 14, 555 (2018)

16. Ahmadi, M. et al.: Observation of the $1 \mathrm{~S}-2 \mathrm{~S}$ transition in trapped antihydrogen. Nature 541, 506 (2017)

17. Ahmadi, M. et al.: Observation of the hyperfine spectrum of antihydrogen. Nature 548, 66 (2017)

18. Gabrielse, G. et al.: Trapped antihydrogen in its ground state. Phys. Rev. Lett. 108, 113002 (2012)

19. Salumbides, E.J., Ubachs, W., Korobov, V.I.: Bounds on fifth forces at the sub- $\AA$ length scale. J. Mol. Spect. 300, 65 (2014)

20. Murata, J., Tanaka, S.: A review of short-range gravity experiments in the LHC era. Class Quantum Grav. 32, 033001 (2015)

21. Brax, P., Fichet, S., Pignol, G.: Bounding quantum dark forces. Phys. Rev. D 97, 115034 (2018)

22. Fichet, S.: Quantum forces from dark matter and where to find them. Phys. Rev. Lett. 120, 131801 (2018)

23. Ficek, F. et al.: Constraints on exotic spin-dependent interactions between matter and antimatter from antiprotonic helium spectroscopy. Phys. Rev. Lett. 120, 183002 (2018)

24. Hori, M., Walz, J.: Physics at CERN's antiproton decelerator. Prog. Part. Nucl. Phys. 72, 206 (2013)

25. Sakimoto, K.: Formation of antiprotonic helium $\bar{p} \mathrm{He}^{+}$and ionization in low-energy collisions of $\bar{p}$ with $\mathrm{He}$ in the ground $1^{1} \mathrm{~S}$ and metastable $2^{3} \mathrm{~S}$ and $2^{1} \mathrm{~S}$ states. Phys. Rev. A 91, 042502 (2015)

26. Korobov, V.I., Zhong, Z.-X., Tian, Q.-L.: Leading term of the He- $\bar{p} \mathrm{He}^{+}$long-range interaction. Phys. Rev. A 92, 052517 (2015)

27. Adamczak, A., Bakalov, D.: Shift and broadening of resonance lines of antiprotonic helium atoms in solid helium. Phys. Rev. A 90, 054501 (2014)

28. Mohr, P.J., Taylor, B.N., Newell, D.B.: CODATA recommended values of the fundamental physical constants: 2010. Rev. Mod. Phys. 84, 1527 (2012)

29. Pohl, R. et al.: The size of the proton. Nature 466, 213 (2010)

30. Crivelli, P., Cooke, D., Heiss, M.W.: Antiproton charge radius. Phys. Rev. D 94, 052008 (2016)

31. Kohno, M., Weise, W.: Proton-antiproton scattering and annihilation into two mesons. Nucl. Phys. A454, 429 (1986)

32. Klempt, E., Bradamante, F., Martin, A., Richard, J.-M.: Antinucleon-nucleon interaction at low energy: scattering and protonium. Phys. Rep. 368, 119 (2002)

33. Friedman, E., Gal, A., Mareš, J.: Antiproton-nucleus potentials from global fits to antiprotonic X-rays and radiochemical data. Nucl. Phys. A761, 283 (2005)

34. Larionov, A.B., Lenske, H.: Elastic scattering, polarization and absorption of relativistic antiprotons on nuclei. Nucl. Phys. A957, 450 (2017)

35. Hori, M. et al.: Determination of the antiproton-to-electron mass ratio by precision laser spectroscopy of $\bar{p} \mathrm{He}^{+}$. Phys. Rev. Lett. 96, 243401 (2006)

36. Hori, M. et al.: Calculation of transition probabilities and ac Stark shifts in two-photon laser transitions of antiprotonic helium. Phys. Rev. A 81, 062508 (2010)

37. Badertscher, A. et al.: Experimental determination of the kinetic energy distribution of $\pi^{-} \mathrm{p}$ atoms in liquid hydrogen. Phys. Lett. B 392, 278 (1997)

38. Aghai-Khozani, H. et al.: First experimental detection of antiproton in-flight annihilation on nuclei at 130 keV. Eur. Phys. J. Plus 127, 125 (2012)

39. Corradini, M. et al.: Experimental apparatus for annihilation cross-section measurements of low energy antiprotons. Nucl. Instrum. Methods A 711, 12 (2013)

40. Sótér, A. et al.: Segmented scintillation detectors with silicon photomultiplier readout for measuring antiproton annihilations. Rev. Sci. Instrum. 85, 023302 (2014)

41. Todoroki, K. et al.: Instrumentation for measurement of in-flight annihilations of $130 \mathrm{keV}$ antiprotons on thin target foils. Nucl. Instrum. Methods A 835, 110 (2016)

42. Aghai-Khozani, H. et al.: Measurement of the antiproton-nucleus annihilation cross-section at low energy. Nucl. Phys. A970, 366 (2018)

43. Hori, M., Dax, A.: Chirp-corrected, nanosecond Ti: Sapphire laser with $6 \mathrm{MHz}$ linewidth for spectroscopy of antiprotonic helium. Opt. Lett. 34, 1273 (2009)

44. Pachucki, K., Czarnecki, A., Jentschura, U.D., Yerokhin, V.A.: Complete two-loop correction to the bound-electron $g$ factor. Phys. Rev. A 72, 022108 (2005)

45. Gabrielse, G. et al.: Precision mass spectroscopy of the antiproton and proton using simultaneously trapped particles. Phys. Rev. Lett. 82, 3198 (1999)

46. Ulmer, S. et al.: High-precision comparison of the antiproton-to-proton charge-to-mass ratio. Nature 524, 196 (2015)

47. Hughes, R.J., Deutch, B.I.: Electric charges of positrons and antiprotons. Phys. Rev. Lett. 69, 578 (1992) 
48. Hori, M., Sótér, A., Korobov, V.I.: Proposed method for laser spectroscopy of pionic helium atoms to determine the charged-pion mass. Phys. Rev. A 89, 042515 (2014)

49. Korobov, V.I., Bakbaev, A.K., Aznabayev, D.T., Zhaugasheva, S.A.: Polarizability of the pionic helium atom. J. Phys. B: At. Mol. Opt. Phys. 48, 245006 (2015)

50. Obreshkov, B., Bakalov, D.: Collisional shift and broadening of the transition lines in pionic helium. Phys. Rev. A 93, 062505 (2016)

51. Trassinelli, M. et al.: Measurement of the charged pion mass using X-ray spectroscopy of exotic atoms. Phys. Lett. B 759, 583 (2016) 